\title{
Validated Determination of NRG1 Ig-like Domain Structure by Mass Spectrometry Coupled with Computational Modeling
}

Niloofar Abolhasani Khaje ${ }^{1}$, Alexander Eletsky², Sarah E. Biehn ${ }^{3}$, Charles K. Mobley ${ }^{1}$, Monique J. Rogals ${ }^{2}$, Yoonkyoo Kim ${ }^{2}$, Sushil K. Mishra ${ }^{1,4}$, Robert J. Doerksen ${ }^{1,4}$, Steffen Lindert ${ }^{3}$, James Prestegard $^{2}$, Joshua S. Sharp ${ }^{1,4,5}$

1. Department of BioMolecular Sciences, University of Mississippi, University, MS 38677

2. Complex Carbohydrate Research Center, University of Georgia, Athens, GA 30602

3. Department of Chemistry and Biochemistry, Ohio State University, Columbus, $\mathrm{OH}$ 43210

4. Glycoscience Center of Research Excellence, University of Mississippi, University, MS 38677

5. Department of Chemistry and Biochemistry, University of Mississippi, University, MS 38677

\footnotetext{
Abstract

High resolution hydroxyl radical protein footprinting (HR-HRPF) is a mass spectrometry-based method that measures the solvent exposure of multiple amino acids in a single experiment, offering constraints for experimentally-informed computational modeling. HR-HRPF-based modeling has previously been used to accurately model the structure of proteins of known structure, but the technique has never been used to determine the structure of a protein of unknown structure leaving questions of unintentional bias and applicability to unknown
} 
structures unresolved. Here, we present the use of HR-HRPF-based modeling to determine the structure of the Ig-like domain of NRG1, a protein with no close homolog of known structure. Independent determination of the protein structure by both HR-HRPF-based modeling and heteronuclear NMR was carried out, with results compared only after both processes were complete. The HR-HRPF-based model was highly similar to the lowest energy NMR model, with a backbone RMSD of 1.6 ̊. To our knowledge, this is the first use of HR-HRPF-based modeling to determine a previously uncharacterized protein structure. 


\section{Introduction}

Mass spectrometry (MS) has rapidly gained in popularity not only in the identification and mass measurement of proteins, but in the characterization of protein higher order structure. Numerous MS-based technologies have been successfully used to characterize protein higher order structure, including hydrogen-deuterium exchange ${ }^{1}$, limited proteolysis ${ }^{2}$, chemical crosslinking ${ }^{3}$ and covalent labeling ${ }^{4}$. Covalent labeling includes a number of techniques, all of which involve reaction of some reagent with amino acid side chains usually available on the surface of the folded protein. A variety of covalent labeling reagents have been used, including acylation reagents ${ }^{5}$, diethylpyrocarbonate ${ }^{6}$, carbenes ${ }^{7}$, trifluoromethyl radicals ${ }^{8,9}$ and iodine radicals ${ }^{10}$. Here, we present an approach based on the use of hydroxyl radicals as a covalent labeling reagent. Hydroxyl radicals generate high quality data for a variety of amino acids, providing a generalizable probe for protein topography ${ }^{4,11-14}$. We also demonstrate that this approach is capable of producing high quality reliable protein structures and validate this in a blind test against a parallel structure determination by NMR methods.

The approach we use begins with data from a technique known as hydroxyl radical protein footprinting (HRPF) ${ }^{15}$. Hydroxyl radicals are useful and popular due to the wide variety of methods for in situ generation ${ }^{16-23}$, broad reactivity ${ }^{13,14}$, small size, hydrophilic nature, and wellcharacterized reaction pathways with various amino acids ${ }^{24}$. Work from Chance and co-workers found that apparent rates of reaction could be correlated with average solvent accessible surface area $(<\mathrm{SASA}\rangle)$ once the inherent rate of reaction of the amino acid was corrected using the free amino acids as a surrogate ${ }^{11,25}$. Work from Sharp and co-workers confirmed these findings, further reporting that amino acids with lower inherent reactivity could display altered inherent 
reactivity based on sequence context ${ }^{12,26}$. Sharp and co-workers further used amino acidresolution HRPF (known as HR-HRPF) coupled with computational modeling to demonstrate the ability to differentiate between accurate computational models and inaccurate computational models, opening possibilities for using HR-HRPF data to determine protein structure ${ }^{12}$.

HR-HRPF data are then used to facilitate computational predictions of structure. The Lindert group developed the first software to use covalent labeling data in automated Rosetta protein structure prediction ${ }^{27,28}$. Recently, Biehn and Lindert reported a more robust and computationally less expensive method for using HR-HRPF data to generate protein models using conical neighbor count instead of $\langle$ SASA $\rangle$, which successfully identified $a b$ initio models of accurate atomic detail for three of the four benchmark proteins examined ${ }^{29}$. However, while these studies indicate the potential of HR-HRPF for the determination of protein structure, no protein of unknown structure has had its structure determined solely using HR-HRPF data to inform computational modeling.

To accurately test the ability of HR-HRPF-based modeling to generate accurate structural models of novel proteins, we used the technology to determine the structure of the immunoglobulin-like domain (NRG1-Ig) of human neuregulin-1 (NRG1). NRG1 is a signaling glycoprotein that interacts with the ErbB/HER family of receptor tyrosine kinases via its EGFlike domain ${ }^{30-32}$. NRG1-mediated signaling plays an important role in neuronal and cardiac development, and regulation of synaptic plasticity ${ }^{31-34}$. Dysregulation of these signaling pathways is implicated in human disease, such as schizophrenia and certain forms of cancer ${ }^{35,36}$. Due to a combination of alternative splicing and proteolytic processing, NRG1 exhibits a high diversity of isoforms, both soluble and membrane-bound, and a number of these isoforms include the Ig-like domain ${ }^{32,37}$. In contrast to the EGF-like domain, the functional role of the 
NRG1-Ig domain is less well understood. It is believed to be involved in binding to heparan sulfate proteoglycans of the extracellular matrix ${ }^{38,39}$, and there are reports that it can affect ErbB receptor activation ${ }^{40-42}$.

In this manuscript, two teams worked independently to characterize the structure of NRG1-Ig. The first team used HR-HRPF to quantitatively measure topography of various amino acid side chains of the NRG1-Ig. Models of the protein were generated via Rosetta ab initio modeling, scored with the HRPF-guided Rosetta score term, then subjected to a Rosetta relaxation ensemble ${ }^{29}$ from which a top scoring model was identified. Meanwhile, the second team determined the structure of NRG1 using standard heteronuclear solution NMR techniques. While all protein was expressed by the second team, no data were shared between groups to prevent any bias in structural characterization. After both teams had generated their structural models, the HR-HRPF constrained structure was compared to the NMR structure, to assess the accuracy of the HR-HRPF method. The results of this study serve as a rigorous and unbiased test of the ability of HR-HRPF to facilitate a reliable determination of soluble protein structures.

\section{Results and Discussion}

\section{HR-HRPF of NRG1-Ig}

Non-glycosylated versions of NRG1-Ig were expressed in E. coli (both with and without isotopic labeling) and purified as described in the Supplementary Information and Figure S1; structural homogeneity was verified by size exclusion chromatography and NMR. For MS studies, proteolytic digestion of NRG1-Ig was optimized for maximum sequence coverage after complete digestion to maximize HR-HRPF data and reproducibility. GluC was found to generate 
considerably higher sequence coverage than trypsin (Figure S2, Supplementary Information), with $98.3 \%$ coverage of the NRG1-Ig expressed sequence. GluC has also successfully been used in the past for HR-HRPF analysis, as the amino acids recognized by GluC are only minor oxidation targets. ${ }^{43}$ Therefore, GluC was used for HR-HRPF analysis.

After purification and digestion optimization, multi-dose Fast Photochemical Oxidation of Proteins (FPOP) ${ }^{12,22,44}$ was performed on NRG1-Ig material at natural isotopic abundance. A mixture of $10 \mu \mathrm{M}$ NRG1-Ig, $17 \mathrm{mM}$ glutamine, $1 \mathrm{mM}$ adenine, $50 \mathrm{mM}$ sodium phosphate, 2.2 $\mathrm{mM}$ Tris (pH 8.1), and hydrogen peroxide at $10 \mathrm{mM}, 25 \mathrm{mM}, 50 \mathrm{mM}$ or $100 \mathrm{mM}$ were used for FPOP labeling. Adenine dosimetry was measured for each experiment to determine delivered radical dose, in order to account for variability in radical generation or scavenging ${ }^{45}$. A control for each FPOP peroxide concentration was conducted under the same conditions without laser irradiation to measure and correct for background oxidation.

Samples were then digested using our optimized GluC protocol. LC-MS/MS using electron transfer dissociation (ETD) was performed to measure the amount of oxidation at each amino acid for each oxidized peptide. Oxidation of twenty amino acids were measured (examples in Figure 1, with full data in Figure S3, Supplementary Information) and converted to the natural $\log$ of a protection factor $(\mathrm{PF})$, defined as normalized relative intrinsic reactivity value for a particular free amino acid residue divided by the regression slope. 95\% confidence intervals for regression slopes were used to represent uncertainty in PF measurement. Values measured for lnPF for all NRG1-Ig amino acids measured are given in Figure S4 and Table S1, Supplementary Information. 


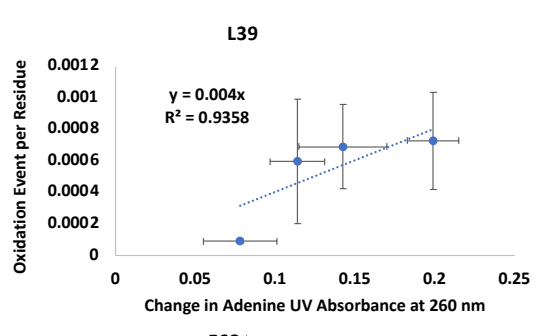

F68 ।

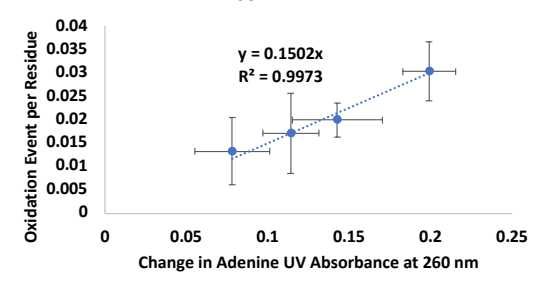

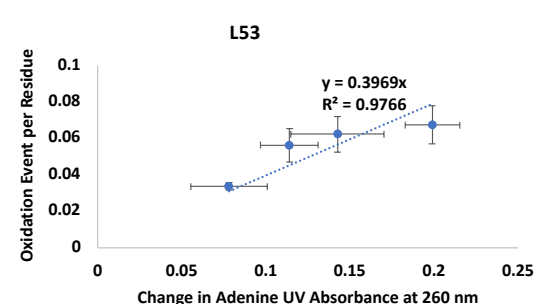

Change in Adenine UV Absorbance at $260 \mathrm{~nm}$

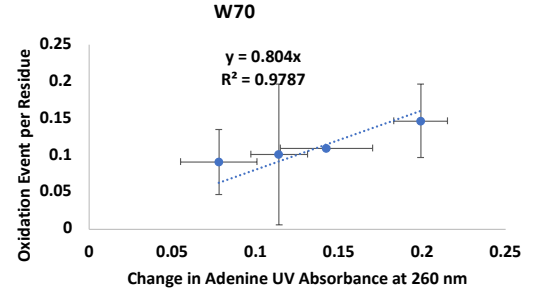

L104

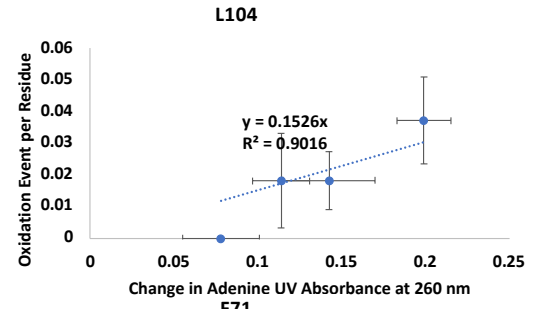

F71

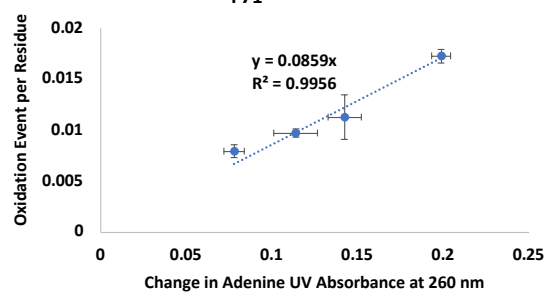

Figure 1. Measured radical dose response rate of 6 amino acids used for structural modeling in NRG1-Ig via HR-HRPF. Each figure shows the calculated oxidation of each residue at 4 different hydrogen peroxide concentrations plotted against the change in adenine absorbance at $260 \mathrm{~nm}$. The error bars represent one standard deviation from triplicate measurements for each data point. Each point represents the oxidation of one residue at a specific radical dose. The slopes of best-fit lines are radical dose responses.

\section{Solution NMR Structure of NRG1-Ig}

Using a suite of standard multidimensional experiments (Table S2, Supplementary Information) we obtained nearly complete resonance assigments of ${ }^{1} \mathrm{H},{ }^{13} \mathrm{C}$ and ${ }^{15} \mathrm{~N}$ spins of the native polypeptide range (Table S3, Fig. S5, Supplementary Information). The only resonances we were unable to observe and assign were those of backbone ${ }^{1} \mathrm{H}$ and ${ }^{15} \mathrm{~N}$ of Lys117. The ${ }^{13} \mathrm{C}^{\alpha}$ and ${ }^{13} \mathrm{C}^{\beta}$ chemical shifts of the two cysteine residues were consistent with disulfide bond formation ${ }^{46}$. Based on extensive chemical shift assignments and NOE data we obtained a welldefined solution NMR structure of NRG1-Ig (Fig. S6, Table S3, Supplementary Information). The fold of NRG1-Ig is typical of immunoglobulin-like domains, with a sandwich of two $\beta$ sheets stabilized by a disulfide bond. The smaller anti-parallel $\beta$-sheet consists of $\beta$-strands 41 58, 94-102, 86-91, while the second $\beta$-sheet consists of $\beta$-strands 77-72, 108-115, 120-130, 45- 
48 in a mixed topology with the last two stands running parallel. The only helical component is a single 310 turn at $104-106$.

\section{Determining the Best Computational Models of NRG1-Ig}

We employed our recent HRPF-guided Rosetta modeling protocol ${ }^{29}$ to predict the structure of NRG1-Ig. As per our published protocol, only lnPF values measured from Trp, Phe, Tyr, His and Leu were used. Our protocol used a HRPF score term, hrf_dynamics, that rewarded models demonstrating agreement with the FPOP labeling data. Additionally, we used Rosetta relaxation ensemble movers to sample protein flexibility. The output structures from the Rosetta mover protocol were referred to as mover models. Upon generation of 20,000 Rosetta $a b$ initio models, we scored models with Rosetta's score function ("Ref15") (Figure 2a) and hrf_dynamics to determine a total score (Figure 2b). The 20 top scoring models were then used as inputs for the relaxation ensemble that generated thirty mover models per top scoring structure, leading to the addition of 600 models to be included in the model distribution (Figure 2c).

Upon examination of the 250 top scoring models when scoring with Rosetta versus scoring with Rosetta and hrf_dynamics including mover models, we observed a decrease in the average root mean squared deviation (RMSD) and an increase in the percentage of models with RMSDs under $5 \AA$ (Figure 2d). The average RMSD of the top 250 models when scoring with Rosetta was $9.5 \AA$, which improved to $3.8 \AA$ when scoring with FPOP data and including mover models. When scoring with Rosetta, $21 \%$ of the top 250 models had RMSDs below $5 \AA$. This improved with hrf_dynamics usage and mover model generation to $94 \%$ of models having RMSDs under 5 A. When scoring both mover models and ab initio structures with our score term, we identified 

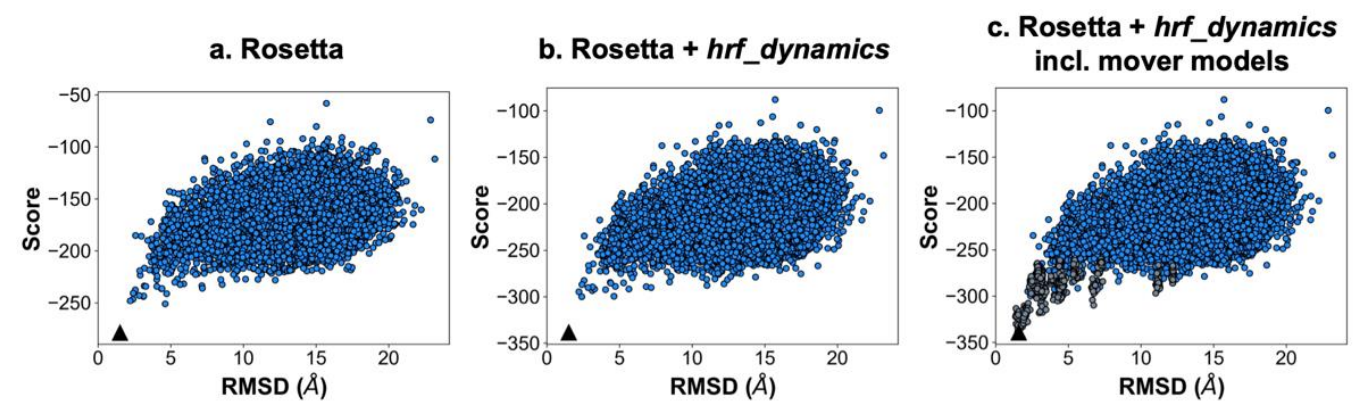

d. RMSD distribution of top 250

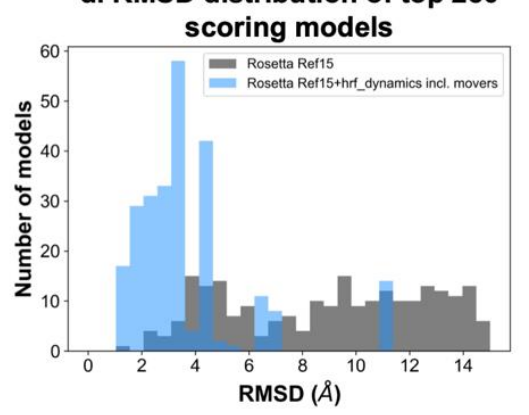

e. Alignment of NMR representative model and best scoring model

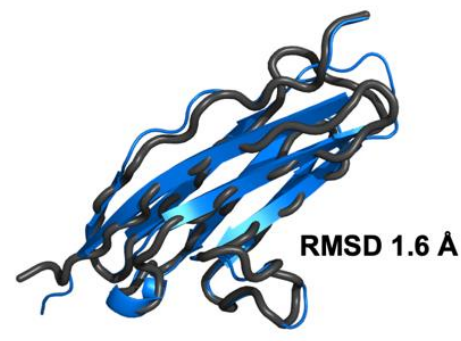

Figure 2. $A b$ initio modeling of NRG1-IG with relaxation ensemble and FPOP-guided scoring significantly enriched high-quality models. Score versus RMSD from NMR model 1 when a, scoring with Rosetta's score function; b, scoring with Rosetta and hrf_dynamics; and c, scoring with Rosetta and hrf_dynamics including mover models (dark grey). Best scoring models are denoted by a black triangle. d, RMSD histograms for top 250 scoring models when scoring with Rosetta (grey) versus Rosetta and hrf_dynamics including mover models (blue). Bin widths were maintained at $0.5 \AA$. e, Alignment of NMR model 1 (black) with the top scoring model identified from our HRPF-guided and mover model protocol (blue). The RMSD to the NMR model was determined to be $1.6 \AA$.

one of the generated mover models as the best scoring model. Our best scoring model exhibited backbone RMSD of 1.6 ̊̊ to the determined NMR structure of NRG1-Ig (Figure 2e).

The use of optimized conical neighbor count (the number of neighbors within the vicinity of a residue based on distance and angle contributions) ${ }^{29}$ to apply FPOP data to computational models is a significant alternative to the use of amino acid solvent accessible surface area data. The correlation between the HR-HRPF lnPF results for NRG1-Ig optimized conical neighbor count from the lowest energy NMR structure was consistent with correlations previously reported for model protein structures ${ }^{11,12,47}$. The subset of amino acids considered here are robust regardless of the method of hydroxyl radical generation or amino acid-level quantitation, 


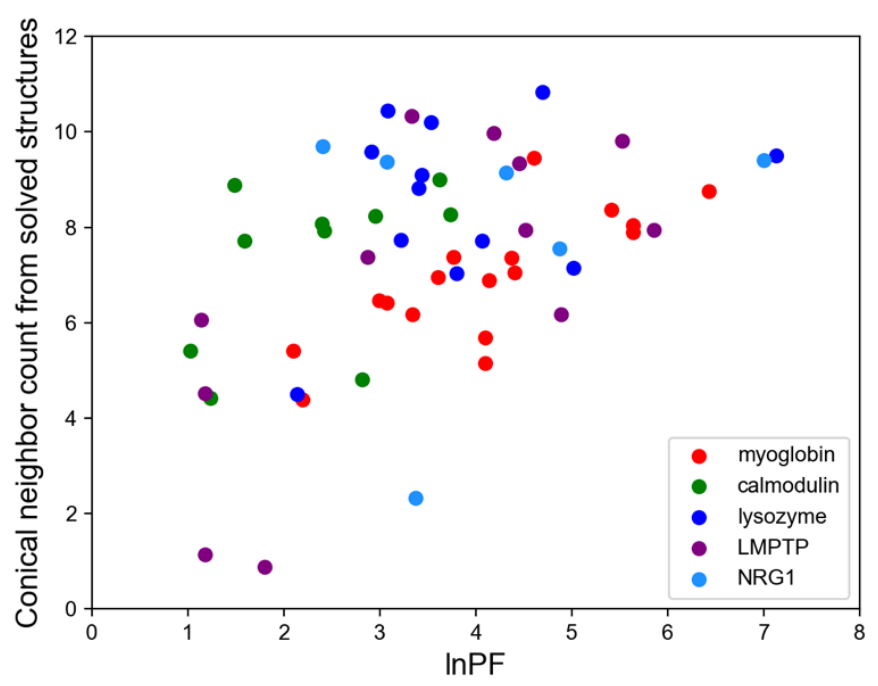

Figure 3. Correlation between HR-HRPF InPF and conical neighbor count. The correlation measured for NRG1 performed blinded to the NMR structure (cyan) was consistent with those reported for proteins of known structure.

and no bias was introduced due to over-fitting to known structures (Figure 3). Overall, employment of the relaxation ensemble to generate mover models resulted in a significant enrichment of accurate, high-quality, low-RMSD models in this blind prediction effort. We concluded that usage of our FPOP-guided and relaxation ensemble method can increase confidence in model selection for other structure prediction efforts.

\section{Conclusion}

In this work, we tested the ability of HR-HRPF combined with conical neighbor count computational modeling to generate accurate, reliable structural models of a protein of truly unknown structure, NRG1-Ig. We were able to greatly increase the reliability of Rosetta modeling by application of HR-HRPF data, generating a final model with a backbone RMSD of $<2 \AA$ from the lowest energy NMR model, and with a large increase in model reliability. As the NRG1-Ig structure was unknown when HR-HRPF was performed and the NMR structure was determined independent of the HR-HRPF group, we have excluded any possibility of 
confirmation bias in our experimental design. The consistency of our results with previous work published on proteins of known structure shown in Figure 3 reveals a lack of confirmation bias in these previous results, and indicates no clear difference in accuracy based on the method of radical generation or amino acid-level oxidation quantification for the subset of amino acids used here (Trp, Phe, Tyr, His and Leu).

Our results as independently confirmed in a blind study by established NMR techniques demonstrate that HR-HRPF combined with conical neighbor count computational modeling is not just a tool for examining relative changes in protein topography, but is a structural biology tool that generates experimentally informed computational models of protein structure that are accurate and reliable. With the rise in computational tools for structural prediction, including the recently released AlphaFold ${ }^{48,49}$, there is a need for flexible experimental methods to validate predicted structures. HR-HRPF has no theoretical limitations on the size or dynamics of measured protein structures, is compatible with protein complexes and can be carried out using microgram quantities of protein. Given the flexibility and low sample requirements of HR-HRPF compared with traditional high-resolution structural biology techniques, this methodology can play a significant role in the validation of computational structures, as well as in the generation of accurate and reliable structural models when computational methods fail. The application of HR-HRPF constraints could also support modern computational methods for predicting structures of protein complexes ${ }^{50}$, which currently have suspected issues regarding comprehensiveness and accuracy in certain cases that could be greatly remedied through the application of experimental HR-HRPF results. Future work examining the ability of HR-HRPF combined with conical neighbor count to correctly identify domain-domain contacts and 
orientation will be important for developing the application of HR-HRPF combined with conical neighbor count to address challenging problems in multi-domain protein structural biology.

\section{Materials and Methods}

\section{Expression \& purification of NRG1-Ig}

A pET-21b(+) plasmid containing a TEV-cleavable N-terminal His-tag and a 100 residue fragment comprising the NRG1-Ig domain (residues 34-133 of the UniProt Q02297 sequence) was purchased from GenScript (US distribution, Piscataway, NJ). This plasmid was transformed into BL21(DE3) E. coli cells (New England Biolabs, Ipswich, MA) using standard protocols. Transformed cells were applied onto LB agar plate with ampicillin followed by overnight incubation at $37^{\circ} \mathrm{C}$. A single colony was used to inoculate a $10 \mathrm{~mL}$ LB media with carbenicillin and incubated overnight at $37^{\circ} \mathrm{C}$. Cells were pelleted at $2000 \mathrm{~g}$ and resuspended in $3 \mathrm{ml}$ of M9 media. Resuspended cells (600 ul) were used to inoculate a $50 \mathrm{ml}$ M9 culture and incubated at 37 C until OD600=0.8.

Glycerol stocks of transformed cells were then used to inoculate $10 \mathrm{~mL}$ of LB starter culture, followed by overnight incubation at $35^{\circ} \mathrm{C}$. Cells were then pelleted and resuspended in $1 \mathrm{~L}$ of LB media, and incubated again at $35^{\circ} \mathrm{C}$. To produce NRG1-Ig at natural isotopic abundance, the $1 \mathrm{~L}$ culture was induced with $1 \mathrm{mM}$ IPTG after reaching $\mathrm{OD}_{600}$ of $\sim 0.6 ; 3 \mathrm{~h}$ after induction cells were harvested by centrifugation at 2,500 $\mathrm{x} g$ and frozen. For stable isotope labeled samples, the 1L LB culture was instead pelleted upon reaching $\mathrm{OD}_{600}$ of $\sim 0.8$, and the cell pellet was resuspended in $0.5 \mathrm{~L}$ of $\mathrm{M} 9$ minimal media containing ${ }^{15} \mathrm{NH}_{3} \mathrm{Cl}$ with either ${ }^{13} \mathrm{C}$-glucose or $5 \%$ ${ }^{13} \mathrm{C}$-glucose (Cambridge Isotope Laboratories). Incubation continued for about $1 \mathrm{hr}$ at $35 \mathrm{C}$ when expression was induced with $1 \mathrm{mM}$ IPTG. Cells were harvested after $\sim 3$ hrs and frozen. 
In both cases, thawed cells were resuspended in lysis buffer $(20 \mathrm{mM}$ Tris $\mathrm{pH} 8.1,300 \mathrm{mM} \mathrm{NaCl}$, and $1 \mathrm{mM}$ TCEP with protease inhibitors) at $4^{\circ} \mathrm{C}$ and lysed using a French-press. The resulting lysate was centrifuged at $12,000 \mathrm{xg}$, and the pellet fraction containing inclusion bodies was resuspended in denaturing buffer $(6 \mathrm{M}$ Urea, 300mM NaCl, $1 \mathrm{mM}$ TCEP, $6 \mathrm{mM}$ imidazole and $20 \mathrm{mM}$ Tris $\mathrm{pH} 8.1$ ) at $4{ }^{\circ} \mathrm{C}$ using either handheld or electric tissue homogenizer. NRG1-Ig was purified under denaturing conditions via immobilized metal affinity chromatography (IMAC) using NGC system (Bio-Rad) equipped with a $10 \mathrm{~mL}$ Co-NTA column. Elution of NRG-Ig1 was accomplished with a linear gradient beginning with 3\% Buffer A (6M Urea, 20mM Tris pH 8.1 at $4{ }^{\circ} \mathrm{C}, 300 \mathrm{mM} \mathrm{NaCl}, 1 \mathrm{mM}$ TCEP) and ending with 100\% Buffer B (6M Urea, 20mM Tris, pH 8.1 at $4{ }^{\circ} \mathrm{C}, 200 \mathrm{mM}$ imidazole, and $\left.300 \mathrm{mM} \mathrm{NaCl}\right)$. The recovered $\mathrm{U}_{-}{ }^{15} \mathrm{~N},{ }^{13} \mathrm{C}, \mathrm{U}-{ }^{15} \mathrm{~N}, 5 \%-{ }^{13} \mathrm{C}$, and natural abundance NRG1-Ig fractions were sealed in in dialysis tubing (Spectrapor, 6-8 kDa) and refolded by dialysis at $4{ }^{\circ} \mathrm{C}$ in four steps against a refolding buffer $(20 \mathrm{mM}$ Tris $\mathrm{pH} 8.1$ at 4 $\left.{ }^{\circ} \mathrm{C}, 300 \mathrm{mM} \mathrm{NaCl}\right)$. The refolding buffer was supplemented with $0.1 \mathrm{mM}$ TCEP and 50uM ethylenediaminetetraacetic acid (EDTA) for the first dialysis stem. $\mathrm{U}_{-}{ }^{15} \mathrm{~N}, 5 \% \mathrm{U}-{ }^{15} \mathrm{~N},{ }^{13} \mathrm{C}$ - and U${ }^{15} \mathrm{~N}, 5 \%-{ }^{13} \mathrm{C}$-labeled NRG1-Ig were subsequently exchanged into NMR buffer (20 mM sodium phosphate $\mathrm{pH} 6.5,100 \mathrm{mM} \mathrm{NaCl}$ ) using $0.5 \mathrm{ml}$ Amicon microconcentrators. $\mathrm{U}-{ }^{15} \mathrm{~N},{ }^{13} \mathrm{C} \mathrm{NMR}$ samples ( $35 \mu \mathrm{l}$ in 1.7 capillary NMR tube), NRG1-Ig NC(I) and NRG1-Ig NC(II), consisted of $0.45 \mathrm{mM}$ and $2.0 \mathrm{mM}$ NRG1-Ig, respectively, with $0.05 \%$ sodium aside, $4 \mu \mathrm{M}$ sodium trimethylsilylpropanesulfonate (DSS) and 7\% $\mathrm{D}_{2} \mathrm{O} . \mathrm{U}-{ }^{15} \mathrm{~N}, 5 \%{ }^{13} \mathrm{C}$ NMR sample, NRG1-Ig NC5 ( $40 \mu \mathrm{l}$ in 1.7 capillary NMR tube), was prepared in the original TRIS refolding buffer, with $0.05 \%$ sodium aside, $5 \mu \mathrm{M}$ sodium trimethylsilylpropanesulfonate (DSS) and $7 \% \mathrm{D}_{2} \mathrm{O}$. For HRPF studies, the NRG1-Ig batch without isotope labeling was subjected to an additional purification stem on a Waters BEH SEC Column, $125 \AA$ A, $1.7 \mu \mathrm{m}, 4.6 \mathrm{~mm} * 300 \mathrm{~mm}$ using a 
Thermo Fisher Dionex 3000 HPLC system. The running buffer was $20 \mathrm{mM}$ Tris at pH 8.1 with $300 \mathrm{mM} \mathrm{NaCl}$ using an isocratic gradient.

Multi-Dose FPOP and NRG1-Ig Digestion

FPOP was performed in triplicate for neuregulin using a 248 nm COMPex Pro 102 high pulse energy excimer $\mathrm{KrF}$ laser in the presence of various hydrogen peroxide concentrations (10 $\mathrm{mM}$, $25 \mathrm{mM}, 50 \mathrm{mM}$, and $100 \mathrm{mM})^{12}$. The experiment was done in triplicate for each hydrogen peroxide concentration. For FPOP, samples were prepared by mixing NRG1-Ig to the final concentration of $10 \mu \mathrm{M}$ in $50 \mathrm{mM}$ sodium phosphate (Sigma-Aldrich), $17 \mathrm{mM}$ glutamine (Acros Organics) and $1 \mathrm{mM}$ adenine (Acros Organics) as a radical dosimeter ${ }^{45}$. Freshly prepared hydrogen peroxide (Fisher Scientific) at 4 different concentrations $(10 \mathrm{mM}, 25 \mathrm{mM}, 50 \mathrm{mM}$, and $100 \mathrm{mM}$ ) was added to each sample prior laser exposure. A total volume of $20 \mu \mathrm{l}$ of sample flowed through the excitation capillary at $17.34 \mathrm{ul} / \mathrm{min}$. The nominal laser fluence at the plane of the excitation capillary was at $9.82 \mathrm{~mJ} / \mathrm{mm}^{2}$ with $15 \%$ exclusion volume. After laser irradiation, the samples were quenched in $25 \mathrm{ul}$ quenching buffer containing $50 \mathrm{nM}$ catalase (SigmaAldrich) and $20 \mathrm{mM}$ methionine amide (Bachem). A control sample for each hydrogen peroxide concentration was done in triplicate with the laser turned off. After laser exposure, we measured the changes in adenine UV absorbance of each oxidized sample as compared to each control at $265 \mathrm{~nm}$ using a nanodrop spectrophotometer. This represents the effective radical dose delivered to the $\operatorname{protein}^{12}$.

After quenching, the oxidized and control samples were denatured and reduced at $95^{\circ} \mathrm{C}$ for 30 minutes in the presence of $5.5 \mathrm{mM}$ DTT (Soltec Ventures). After denaturation, the samples were put on ice for 2 minutes. More sodium phosphate buffer at $\mathrm{pH} 6$ was added to keep its 
concentration at $30 \mathrm{mM}$ prior to GluC addition. GluC (Promega Corp) was added in 1:20

enzyme: protein mass ratio. The samples were digested overnight for 14 hours.

\section{C18 RPLC-MS/MS C18}

LC-MS/MS was done using an Acclaim PepMap 100 C18 nanocolumn $(0.075 \mathrm{~mm} \times 150 \mathrm{~mm}$, $2 \mu \mathrm{m}$ particle size, $100 \AA$ pore size, Thermo Fisher Scientific) coupled to a $300 \mu \mathrm{m}$ i.d. $\times 5 \mathrm{~mm}$ C18 PepMap 100 trap column with $5 \mu \mathrm{m}$ particle size (Thermo Fisher Scientific) to desalt and concentrate the samples before loading onto the C18 nanocolumn for separation. LC-MS buffers were made using all LC/MS grade solvents (Fisher Scientific). The capillary pump was used to load the samples onto the C18 trap column using buffer $\mathrm{C}$ (water $+0.05 \% \mathrm{TFA}$ ) and buffer D (acetonitrile $+0.05 \%$ TFA). We used a nanopump for chromatographic separation using mobile phase $\mathrm{E}$ (water+ $0.1 \%$ formic acid) and mobile phase $\mathrm{F}$ (acetonitrile+ $0.1 \%$ formic acid). Initially, the samples were loaded onto the C18 trap column in $98 \% \mathrm{C}, 2 \% \mathrm{D}$ at $5 \mu \mathrm{l} / \mathrm{min}$ for 6 minutes. The trap column was then switched inline with the nanocolumn and trapped peptides were back-eluted onto the nanocolumn using the nanopump. Elution started by increasing buffer $\mathrm{F}$ in a linear gradient from $2 \%$ to $40 \%$ (with the balance as buffer $\mathrm{E}$ ) over 22 minutes. The gradient was then ramped up to $95 \% \mathrm{~F}$ over 5 minutes and held isocratic for 3 minutes to wash the column. Buffer $\mathrm{F}$ was then decreased to $2 \%$ over 1 minute and held isocratic for 6 minutes to re-equilibrate the column for the next run. The samples were eluted directly into a nanospray source of a Thermo Fusion Tribrid orbitrap, where the spray voltage was set at $2600 \mathrm{~V}$ and ion transfer tube temperature at $300{ }^{\circ} \mathrm{C}$. A full MS scan was obtained from 150 to $2000 \mathrm{~m} / \mathrm{z}$. CID and ETD was performed every 2 seconds on precursor ions of +2 charge and greater for peptide identification and sequence coverage analysis. For ions with +2 charge state, ETD was performed with $20 \%$ EThcD SA collision energy to increase ETD fragmentation. The orbitrap 
resolution for both ETD and EThcD was 30000 with Automatic Gain Control target at 5e4 and maximum injection time of $100 \mathrm{~ms}$.

Peptide and Amino Acid level Oxidation Analysis

Byonic version v2.10.5 (Protein Metrics) was used to identify NRG1-Ig peptide sequences using the NRG1-Ig protein sequence described in Figure S2 (Supplementary Information). For all peptides detected, the major oxidation products detected were net additions of one or more oxygen atoms. In order to calculate average oxidation events per peptide, the area under the curve for peaks of unoxidized and oxidized peptides was used according to Equation 1. Briefly, the oxidation events per peptide were calculated by summing the intensity $(I)$ of each peptide oxidation product multiplied by the number of oxidation events on the peptide required to generate that product and divided by the sum of $I$ for all oxidized and unoxidized versions of that peptide, as shown in Equation 1. $P$ represents the average oxidation events per peptide, and $I$ is the area under the curve for peaks of oxidized and unoxidized peptides.

\section{Equation 1.}

$$
P=\left[\frac{I \text { singly oxidized } * 1+I \text { doubly oxidized } * 2+I \text { triply oxidized } * 3+\cdots}{\text { I singly oxidized }+ \text { I doubly oxidized }+ \text { I triply oxidized }+\cdots}\right]
$$

The amount of oxidation at residue level quantitation in a peptide was determined by the fragment ion ( $\mathrm{z}$ or $\mathrm{c}$ ion) intensities of the peptide ETD fragmentation. The oxidation fraction of a given $\mathrm{z}$ or $\mathrm{c}$ ion was calculated by dividing the oxidized sequence ion intensity by the sum of the intensity of the corresponding oxidized and unoxidized sequence ion in a particular oxidized peptide. The relative oxidation fraction of each product ion $f\left(\mathrm{Z}_{\mathrm{i}}\right)$ was calculated using Equation 3.2 where $\mathrm{I}\left(\mathrm{Z}_{\mathrm{i}}\right)$ is the intensity of the designated product ion summed across all spectra. 


\section{Equation 2.}

$$
f(Z i) \text { relative }=\frac{I(Z i) \text { oxidized }}{(I(Z i) \text { oxidized }+I(Z i) \text { unoxidized })}
$$

The absolute amount of oxidation of a given amino acid was determined by multiplying the average oxidation event of a peptide by the absolute fractional oxidation of the corresponding sequence ions. As shown in Equation 3, $P$ is the average oxidation event per peptide calculated from Equation 1, and the term in brackets is the fractional difference of two adjacent sequence ions, $\mathrm{f}\left(\mathrm{Z}_{\mathrm{i}}\right)$ and $\mathrm{f}\left(\mathrm{Z}_{\mathrm{i}-1}\right)$. In cases where ETD fragmentation ions are not adjacent in sequence, fractional oxidation for multiple contiguous residues within the peptide was calculated by using non-adjacent ETD fragments in Equation 3.

\section{Equation 3.}

$$
\text { Oxidation event per amino acid }=\left[f\left(Z_{i}\right)-f\left(Z_{i-1}\right)\right] * P
$$

In order to take background oxidation into account, the oxidation event of each residue was calculated by subtracting the oxidation event of the same residue under control conditions from its oxidation event in the oxidized sample.

Natural Log Protection Factor $(\ln (\mathrm{PF}))$ was calculated using Equation 4 where $R_{i}$ represents the amino acid intrinsic reactivity for residue $i$ while $S l o p e_{i}$ represents the experimentally determined radical dose response for residue $i$.

\section{Equation 4.}

$$
\ln \left(P F_{i}\right)=\ln \left(\frac{R_{i}}{\operatorname{Slope}_{i}}\right)
$$




\section{Structural Modeling}

Using Rosetta's AbInitioRelax protocol, the sequence of NRG1-Ig (adapted from UniProt Q02297), and fragment libraries obtained from the Robetta server, 20,000 ab initio models of NRG1-Ig were built. ${ }^{51-55}$ No FPOP data were included during model generation. Models were scored with the "Ref15" Rosetta score function. Per-residue FPOP data were converted into the natural $\log$ of the protection factor $(\ln P F) .{ }^{25,28,29}$ The $\operatorname{lnPF}$ values were supplied to the hrf_dynamics term, and models were scored based on their agreement with the labeling data. ${ }^{29}$ The summed per-residue hrf_dynamics score used a weight of 9.0, as described previously. The total score was determined by adding the Rosetta and hrf_dynamics scores. Models were ranked by total score. The twenty top-scoring models were then used as input for mover model generation with the Rosetta relaxation ensemble, as described previously. ${ }^{29}$ For each of the topscoring structures, thirty mover models were obtained. The six hundred mover models were scored with Rosetta and hrf_dynamics and then included in the ab initio model distribution. The best scoring model was selected as our blind prediction for the NRG1-Ig structure, for comparison with the NMR structure. For comparison between HR-HRPF and NMR structural models, C $\alpha$ RMSD values with no outlier rejection were calculated with Rosetta.

\section{NRG1-Ig NMR Spectroscopy and Structure Calculation}

NMR data collection, processing, resonance assignment, and structure calculation followed the protocols of Northeast Structural Genomics Consortium (NESG Wiki, http://www.nmr2.buffalo.edu/nesg.wiki/Main_Page). NMR spectra (Table S2, Supplementary Information) for NRG1-Ig samples were acquired at $25^{\circ} \mathrm{C}$ on an AVANCE NEO $800 \mathrm{MHz}$ spectrometer (Bruker BioSpin) equipped with a $1.7 \mathrm{~mm} \mathrm{TCI}{ }^{1} \mathrm{H}\left({ }^{13} \mathrm{C},{ }^{15} \mathrm{~N}\right)$ cryogenic probe. All spectra were Fourier-transformed using Topspin v4 (Bruker Biospin), except non-uniformly 
sampled 3D HBHA(CO)NH, which was reconstructed using Smile ${ }^{56}$ and Fourier-transformed with NMRPipe ${ }^{57} .{ }^{1} \mathrm{H}$ chemical shifts were referenced relative to 4,4-dimethyl-4-silapentane-1sulfonic acid (DSS), and ${ }^{13} \mathrm{C}$ and ${ }^{15} \mathrm{~N}$ chemical shifts were referenced indirectly via gyromagnetic ratios. Visualization and analysis of NMR spectra, NOE peak picking and integration were performed with the program CARA ${ }^{58}$. Automated assignment of backbone ${ }^{1} \mathrm{H},{ }^{15} \mathrm{~N},{ }^{13} \mathrm{CO},{ }^{13} \mathrm{C}^{\alpha}$ and ${ }^{13} \mathrm{C}^{\beta}$ resonances was obtained with AutoAssign ${ }^{59}$ followed by interactive validation and completion. Side-chain resonances were assigned interactively using 3D $(\mathrm{H}) \mathrm{CCH}$ and 3D ${ }^{13} \mathrm{C} /{ }^{15} \mathrm{~N}$-edited $\left[{ }^{1} \mathrm{H},{ }^{1} \mathrm{H}\right]$ NOESY spectra. Stereospecific assignments of Leu and Val isopropyl groups were obtained based on positive versus negative peak intensities in the $2 \mathrm{D}\left[{ }^{13} \mathrm{C},{ }^{1} \mathrm{H}\right]$ constant-time HSQC (CT-HSQC) acquired for NRG1-Ig NC5, as described previously ${ }^{60}$. Stereospecific assignment of Asn and $\mathrm{Gln} \mathrm{CONH}_{2}$ groups were determined from relative NOE peak intensities.

Structure calculation and automatic NOE peak assignment was performed iteratively using CYANA v 3.98.13 ${ }^{61,62}$ and ASDP v1.0 ${ }^{63}$. Constraints for backbone $\varphi, \psi$ and side-chain $\chi_{1}$ dihedral angles were derived using TALOS-N ${ }^{64}$, and those that were consistent with the initial structural models were used in subsequent structure calculation steps. NOE peaks with matching unambiguous assignments from CYANA and ASDP were manually checked and refined for consistency with NOE spectra and distance constraint violations, and then used to optimize NOE distance calibration function. Assignments of these peaks were kept fixed during final structure calculation with CYANA. Stereospecific assignment of $\mathrm{CH}_{2}$ groups was performed iteratively using the GLOMSA module of CYANA. Of 100 calculated conformers, 20 conformers with the lowest target function values were further refined in explicit water bath using CNS ${ }^{65}$ as previously described ${ }^{66}$ with distance constraints relaxed by $5 \%$. The quality of NRG1-Ig 
structure models was analyzed with PSVS ${ }^{67}$, and the resulting statistics are summarized in

Table S3, Supplementary Information. Software used for NMR data analysis and structure calculation was accessed via NMRBox ${ }^{68}$. Atomic coordinates, structural restraints, assigned NMR chemical shifts and NOE peaklists were deposited in the Protein Data Bank (PDB ID 7SJL) and BioMagResBank (accession code 30960).

\section{Acknowledgements}

This work was supported by the National Institute of General Medical Sciences (R01GM127267). S.M. and R.J.D. acknowledge support from the Glycoscience Center of Research Excellence (NIH P20GM130460) and J.H.P. acknowledges support from (R01GM033225). This study made use of NMRbox: National Center for Biomolecular NMR Data Processing and Analysis, a Biomedical Technology Research Resource (BTRR), which is supported by NIH grant P41GM111135 (NIGMS). Modeling work in this publication was supported by NSF (CHE 1750666) to S.L.

\section{Conflict of Interest Disclosure}

J.S.S. discloses a significant financial interest in GenNext Technologies, Inc., a company commercializing technologies for protein higher order structure analysis.

\section{References}

1 Engen, J. R., Botzanowski, T., Peterle, D., Georgescauld, F. \& Wales, T. E. Developments in Hydrogen/Deuterium Exchange Mass Spectrometry. Anal Chem 93, 567-582, doi:10.1021/acs.analchem.0c04281 (2021).

2 Feng, Y. et al. Global analysis of protein structural changes in complex proteomes. Nat Biotechnol 32, 1036-1044, doi:10.1038/nbt.2999 (2014). 
3 Yugandhar, K., Zhao, Q., Gupta, S., Xiong, D. \& Yu, H. Progress in methodologies and quality-control strategies in protein cross-linking mass spectrometry. Proteomics, e2100145, doi:10.1002/pmic.202100145 (2021).

4 Liu, X. R., Zhang, M. M. \& Gross, M. L. Mass Spectrometry-Based Protein Footprinting for Higher-Order Structure Analysis: Fundamentals and Applications. Chem Rev 120, 4355-4454, doi:10.1021/acs.chemrev.9b00815 (2020).

5 Suckau, D., Mak, M. \& Przybylski, M. Protein surface topology-probing by selective chemical modification and mass spectrometric peptide mapping. Proc Natl Acad Sci U S A 89, 5630-5634 (1992).

6 Pan, X., Limpikirati, P., Chen, H., Liu, T. \& Vachet, R. W. Higher-Order Structure Influences the Kinetics of Diethylpyrocarbonate Covalent Labeling of Proteins. J Am Soc Mass Spectrom 31, 658-665, doi:10.1021/jasms.9b00132 (2020).

7 Jumper, C. C., Bomgarden, R., Rogers, J., Etienne, C. \& Schriemer, D. C. Highresolution mapping of carbene-based protein footprints. Anal Chem 84, 4411-4418, doi:10.1021/ac300120z (2012).

8 Cheng, M., Zhang, B., Cui, W. \& Gross, M. L. Laser-Initiated Radical Trifluoromethylation of Peptides and Proteins: Application to Mass-Spectrometry-Based Protein Footprinting. Angewandte Chemie (International ed 56, 14007-14010, doi:10.1002/anie.201706697 (2017).

9 Cheng, M. et al. Fast Protein Footprinting by X-ray Mediated Radical Trifluoromethylation. J Am Soc Mass Spectrom 31, 1019-1024, doi:10.1021/jasms.0c00085 (2020). 
10 Cheng, M., Guo, C., Li, W. \& Gross, M. L. Free-Radical Membrane Protein Footprinting by Photolysis of Perfluoroisopropyl Iodide Partitioned to Detergent Micelle by Sonication. Angewandte Chemie (International ed 60, 8867-8873, doi:10.1002/anie.202014096 (2021).

11 Kaur, P., Kiselar, J., Yang, S. \& Chance, M. R. Quantitative protein topography analysis and high-resolution structure prediction using hydroxyl radical labeling and tandem-ion mass spectrometry (MS). Mol Cell Proteomics 14, 1159-1168, doi:10.1074/mcp.O114.044362 (2015).

12 Xie, B., Sood, A., Woods, R. J. \& Sharp, J. S. Quantitative Protein Topography Measurements by High Resolution Hydroxyl Radical Protein Footprinting Enable Accurate Molecular Model Selection. Sci Rep 7, 4552, doi:10.1038/s41598-017-04689-3 (2017).

13 Buxton, G. V., Greenstock, C. L., Helman, W. P. \& Ross, A. B. Critical-Review of Rate Constants for Reactions of Hydrated Electrons, Hydrogen-Atoms and Hydroxyl Radicals (.OH/.O-) in Aqueous-Solution. J Phys Chem Ref Data 17, 513-886 (1988).

$14 \mathrm{Xu}, \mathrm{G}$. \& Chance, M. R. Radiolytic modification and reactivity of amino acid residues serving as structural probes for protein footprinting. Anal Chem 77, 4549-4555 (2005).

15 McKenzie-Coe, A., Montes, N. S. \& Jones, L. M. Hydroxyl Radical Protein Footprinting: A Mass Spectrometry-Based Structural Method for Studying the Higher Order Structure of Proteins. Chem Rev, doi:10.1021/acs.chemrev.1c00432 (2021).

16 Sharp, J. S., Becker, J. M. \& Hettich, R. L. Protein surface mapping by chemical oxidation: structural analysis by mass spectrometry. Anal Biochem 313, 216-225 (2003). 
17 Sharp, J. S., Becker, J. M. \& Hettich, R. L. Analysis of protein solvent accessible surfaces by photochemical oxidation and mass spectrometry. Anal Chem 76, 672-683 (2004).

18 Sharp, J. S. et al. Photochemical surface mapping of C14S-Sml1p for constrained computational modeling of protein structure. Anal Biochem 340, 201-212 (2005).

19 Maleknia, S. D., Chance, M. R. \& Downard, K. M. Electrospray-assisted modification of proteins: a radical probe of protein structure. Rapid Commun Mass Spectrom 13, 23522358 (1999).

20 Maleknia, S. D. \& Downard, K. M. Unfolding of apomyoglobin helices by synchrotron radiolysis and mass spectrometry. Eur J Biochem 268, 5578-5588 (2001).

21 Aye, T. T., Low, T. Y. \& Sze, S. K. Nanosecond laser-induced photochemical oxidation method for protein surface mapping with mass spectrometry. Anal Chem 77, 5814-5822 (2005).

22 Hambly, D. M. \& Gross, M. L. Laser flash photolysis of hydrogen peroxide to oxidize protein solvent-accessible residues on the microsecond timescale. J Am Soc Mass Spectrom 16, 2057-2063 (2005).

23 Minkoff, B. B. et al. Plasma-Generated OH Radical Production for Analyzing ThreeDimensional Structure in Protein Therapeutics. Sci Rep 7, 12946, doi:10.1038/s41598017-13371-7 (2017).

24 Garrison, W. M. Reaction Mechanisms in the Radiolysis of Peptides, Polypeptides, and Proteins. Chem Rev 87, 381-398 (1987).

25 Huang, W., Ravikumar, K. M., Chance, M. R. \& Yang, S. Quantitative mapping of protein structure by hydroxyl radical footprinting-mediated structural mass spectrometry: 
a protection factor analysis. Biophys $J \mathbf{1 0 8}, 107-115$, doi:10.1016/j.bpj.2014.11.013 (2015).

26 Sharp, J. S. \& Tomer, K. B. Effects of anion proximity in peptide primary sequence on the rate and mechanism of leucine oxidation. Anal Chem 78, 4885-4893 (2006).

27 Aprahamian, M. L. \& Lindert, S. Utility of Covalent Labeling Mass Spectrometry Data in Protein Structure Prediction with Rosetta. J Chem Theory Comput 15, 3410-3424, doi:10.1021/acs.jctc.9b00101 (2019).

28 Aprahamian, M. L., Chea, E. E., Jones, L. M. \& Lindert, S. Rosetta Protein Structure Prediction from Hydroxyl Radical Protein Footprinting Mass Spectrometry Data. Anal Chem 90, 7721-7729, doi:10.1021/acs.analchem.8b01624 (2018).

29 Biehn, S. E. \& Lindert, S. Accurate protein structure prediction with hydroxyl radical protein footprinting data. Nat Commun 12, 341, doi:10.1038/s41467-020-20549-7 (2021).

30 Liu, P. et al. A single ligand is sufficient to activate EGFR dimers. Proc Natl Acad Sci U S A 109, 10861-10866, doi:10.1073/pnas.1201114109 (2012).

31 Mei, L. \& Nave, K. A. Neuregulin-ERBB signaling in the nervous system and neuropsychiatric diseases. Neuron 83, 27-49, doi:10.1016/j.neuron.2014.06.007 (2014).

32 Mei, L. \& Xiong, W. C. Neuregulin 1 in neural development, synaptic plasticity and schizophrenia. Nat Rev Neurosci 9, 437-452, doi:10.1038/nrn2392 (2008).

33 Xu, Y., Li, X., Liu, X. \& Zhou, M. Neuregulin-1/ErbB signaling and chronic heart failure. Adv Pharmacol 59, 31-51, doi:10.1016/S1054-3589(10)59002-1 (2010).

34 Kataria, H., Alizadeh, A. \& Karimi-Abdolrezaee, S. Neuregulin-1/ErbB network: An emerging modulator of nervous system injury and repair. Prog Neurobiol 180, 101643, doi:10.1016/j.pneurobio.2019.101643 (2019). 
35 Shi, L. \& Bergson, C. M. Neuregulin 1: an intriguing therapeutic target for neurodevelopmental disorders. Transl Psychiatry 10, 190, doi:10.1038/s41398-02000868-5 (2020).

36 Laskin, J. et al. NRG1 fusion-driven tumors: biology, detection, and the therapeutic role of afatinib and other ErbB-targeting agents. Ann Oncol 31, 1693-1703, doi:10.1016/j.annonc.2020.08.2335 (2020).

37 Willem, M. Proteolytic processing of Neuregulin-1. Brain Res Bull 126, 178-182, doi:10.1016/j.brainresbull.2016.07.003 (2016).

38 Pankonin, M. S., Gallagher, J. T. \& Loeb, J. A. Specific structural features of heparan sulfate proteoglycans potentiate neuregulin-1 signaling. J Biol Chem 280, 383-388, doi:10.1074/jbc.M402645200 (2005).

39 Loeb, J. A. \& Fischbach, G. D. ARIA can be released from extracellular matrix through cleavage of a heparin-binding domain. J Cell Biol 130, 127-135, doi:10.1083/jcb.130.1.127 (1995).

40 Li, Q. \& Loeb, J. A. Neuregulin-heparan-sulfate proteoglycan interactions produce sustained erbB receptor activation required for the induction of acetylcholine receptors in muscle. J Biol Chem 276, 38068-38075, doi:10.1074/jbc.M104485200 (2001).

41 Centa, A., Rodriguez-Barrueco, R., Montero, J. C. \& Pandiella, A. The immunoglobulinlike domain of neuregulins potentiates ErbB3/HER3 activation and cellular proliferation. Mol Oncol 12, 1061-1076, doi:10.1002/1878-0261.12310 (2018).

42 Eto, K., Eda, K., Kanemoto, S. \& Abe, S. The immunoglobulin-like domain is involved in interaction of Neuregulin1 with ErbB. Biochem Biophys Res Commun 350, 263-271, doi:10.1016/j.bbrc.2006.09.028 (2006). 
43 Li, Z. et al. High structural resolution hydroxyl radical protein footprinting reveals an extended Robo1-heparin binding interface. J Biol Chem 290, 10729-10740, doi:10.1074/jbc.M115.648410 (2015).

44 Gau, B. C., Sharp, J. S., Rempel, D. L. \& Gross, M. L. Fast photochemical oxidation of protein footprints faster than protein unfolding. Anal Chem 81, 6563-6571, doi:10.1021/ac901054w (2009).

45 Xie, B. \& Sharp, J. S. Hydroxyl Radical Dosimetry for High Flux Hydroxyl Radical Protein Footprinting Applications Using a Simple Optical Detection Method. Anal Chem 87, 10719-10723, doi:10.1021/acs.analchem.5b02865 (2015).

46 Sharma, D. \& Rajarathnam, K. 13C NMR chemical shifts can predict disulfide bond formation. J Biomol NMR 18, 165-171, doi:10.1023/a:1008398416292 (2000).

47 Stanford, S. M. et al. Diabetes reversal by inhibition of the low-molecular-weight tyrosine phosphatase. Nat Chem Biol 13, 624-632, doi:10.1038/nchembio.2344 (2017).

48 AlQuraishi, M. AlphaFold at CASP13. Bioinformatics (Oxford, England) 35, 4862-4865, doi:10.1093/bioinformatics/btz422 (2019).

49 Jumper, J. et al. Highly accurate protein structure prediction with AlphaFold. Nature 596, 583-589, doi:10.1038/s41586-021-03819-2 (2021).

50 Humphreys, I. R. et al. Computed structures of core eukaryotic protein complexes. Science 374, eabm4805, doi:10.1126/science.abm4805 (2021).

51 Bender, B. J. et al. Protocols for molecular modeling with Rosetta3 and RosettaScripts. Biochemistry 55, 4748-4763 (2016).

52 Alford, R. F. et al. The Rosetta all-atom energy function for macromolecular modeling and design. Journal of Chemical Theory and Computation 13, 3031-3048 (2017). 
53 Kim, D. E., Chivian, D. \& Baker, D. Protein structure prediction and analysis using the Robetta server. Nucleic Acids Research 32, W526-W531 (2004).

54 Leman, J. K. et al. Macromolecular modeling and design in Rosetta: recent methods and frameworks. Nature Methods, 1-14 (2020).

55 Biehn, S. E. \& Lindert, S. Protein Structure Prediction with Mass Spectrometry Data. Annual review of physical chemistry, doi:10.1146/annurev-physchem-082720-123928 (2021).

56 Ying, J., Delaglio, F., Torchia, D. A. \& Bax, A. Sparse multidimensional iterative lineshape-enhanced (SMILE) reconstruction of both non-uniformly sampled and conventional NMR data. J Biomol NMR 68, 101-118, doi:10.1007/s10858-016-0072-7 (2017).

57 Delaglio, F. et al. NMRPipe: a multidimensional spectral processing system based on UNIX pipes. J Biomol NMR 6, 277-293, doi:10.1007/BF00197809 (1995).

58 Keller, R. L. J. The Computer Aided Resonance Assignment Tutorial. (CANTINA Verlag, 2004).

59 Zimmerman, D. E. et al. Automated analysis of protein NMR assignments using methods from artificial intelligence. J Mol Biol 269, 592-610, doi:10.1006/jmbi.1997.1052 (1997).

60 Neri, D., Szyperski, T., Otting, G., Senn, H. \& Wuthrich, K. Stereospecific nuclear magnetic resonance assignments of the methyl groups of valine and leucine in the DNAbinding domain of the 434 repressor by biosynthetically directed fractional 13C labeling. Biochemistry 28, 7510-7516, doi:10.1021/bi00445a003 (1989). 
61 Guntert, P., Mumenthaler, C. \& Wuthrich, K. Torsion angle dynamics for NMR structure calculation with the new program DYANA. J Mol Biol 273, 283-298, doi:10.1006/jmbi.1997.1284 (1997).

62 Herrmann, T., Guntert, P. \& Wuthrich, K. Protein NMR structure determination with automated NOE assignment using the new software CANDID and the torsion angle dynamics algorithm DYANA. J Mol Biol 319, 209-227, doi:10.1016/s00222836(02)00241-3 (2002).

63 Huang, Y. J., Mao, B., Xu, F. \& Montelione, G. T. Guiding automated NMR structure determination using a global optimization metric, the NMR DP score. J Biomol NMR 62 , 439-451, doi:10.1007/s10858-015-9955-2 (2015).

64 Shen, Y. \& Bax, A. Protein backbone and sidechain torsion angles predicted from NMR chemical shifts using artificial neural networks. J Biomol NMR 56, 227-241, doi:10.1007/s10858-013-9741-y (2013).

65 Brunger, A. T. Version 1.2 of the Crystallography and NMR system. Nature protocols 2 , 2728-2733, doi:10.1038/nprot.2007.406 (2007).

66 Linge, J. P., Williams, M. A., Spronk, C. A., Bonvin, A. M. \& Nilges, M. Refinement of protein structures in explicit solvent. Proteins 50, 496-506, doi:10.1002/prot.10299 (2003).

67 Bhattacharya, A., Tejero, R. \& Montelione, G. T. Evaluating protein structures determined by structural genomics consortia. Proteins 66, 778-795, doi:10.1002/prot.21165 (2007).

68 Maciejewski, M. W. et al. NMRbox: A Resource for Biomolecular NMR Computation. Biophys J 112, 1529-1534, doi:10.1016/j.bpj.2017.03.011 (2017). 
bioRxiv preprint doi: https://doi.org/10.1101/2021.12.13.472484; this version posted December 14,2021 . The copyright holder for this preprint (which was not certified by peer review) is the author/funder, who has granted bioRxiv a license to display the preprint in perpetuity. It is made available under aCC-BY-NC-ND 4.0 International license. 